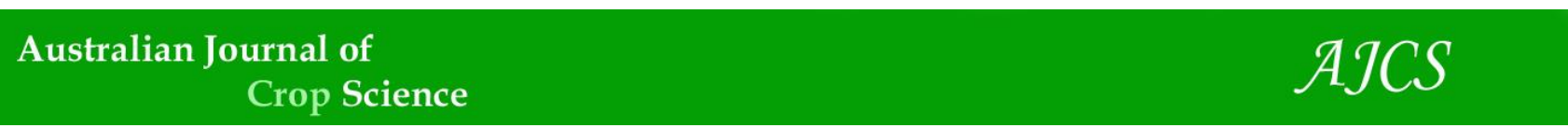

AJCS 15(04):494-504 (2021)

ISSN:1835-2707

doi: 10.21475/ajcs.21.15.04.p2676

\title{
High Robusta coffee plant density is associated with better yield potential at mixed responses for growth robustness, pests and diseases: which way for a farmer?
}

\author{
Godfrey Sseremba*, Godfrey Hubby Kagezi, Judith Kobusinge, Pascal Musoli, David Akodi, Nicholas \\ Olango, Patrick Kucel, Job Chemutai, Joseph Mulindwa, Geofrey Arinaitwe
}

National Coffee Research Institute, National Agricultural Research Organization, P.O. Box 185, Mukono, Uganda

*Corresponding author: gsseremba16@gmail.com

\begin{abstract}
Plant density in Robusta coffee is an unresolved issue in low volume producing countries especially when compared with leading producers. In this study, we aimed to compare the response of Robusta coffee to pest incidence, disease severity, growth and yield potential in two contrasting spacing regimes. Two spacing regimes of $3 \mathrm{~m} \times 3 \mathrm{~m}$ and $3 \mathrm{~m} \times 1 \mathrm{~m}$ were evaluated for selected parameters in a randomized complete block design with three replications. There was a highly significant difference in pest incidence between the spacing regimes $(p<0.01)$ for all the pests except scales $(p=0.126)$. The black coffee trig borer incidence was higher under close spacing of $3 \mathrm{~m} \times 1 \mathrm{~m}$ than for $3 \mathrm{~m} \times 3 \mathrm{~m}$ with a mean difference of $13.2 \%$. There was no significant association between spacing regime and leaf rust disease incidence while the association was significant for red blister disease $(\chi 2=33.56, d f=1, p<0.001)$. Significant difference in growth response between spacing regimes $(p<0.05)$ were also obtained for change in canopy height (dCAH), number of primaries, number of stems and leaf size. For instance, dCAH was higher under $3 \mathrm{~m} \times 1 \mathrm{~m}$ spacing than for $3 \mathrm{~m} \times 3 \mathrm{~m}$ spacing. A significant difference in yield potential existed between the spacing regimes $(p<0.05)$ for average yield per tree and average yield per hectare $(\mathrm{aYH})$. Close spacing produced a higher aYH $(5.82 \mathrm{t} \mathrm{cc} / \mathrm{ha})$ than wide spacing $(4.80 \mathrm{t} \mathrm{cc} / \mathrm{ha})$. Whereas yield potential is high at high tree densities, associated prevalence of biotic constraints calls for supportive stress management package for farmers.
\end{abstract}

Keywords: Recommended spacing; Robusta coffee productivity; plant population; Black coffee trig borer incidence; Disease severity.

Abbreviations: BCTB_Black coffee trig borer; CBB_Coffee berry borer; CLR_Coffee leaf rust; FAO_Food and Agriculture Organization of the United Nations; GoU_Government of Uganda; kg cc/ha_kilograms of clean coffee per tree.

\section{Introduction}

Coffee (Coffea spp.) is the main traditional cash crop in Uganda, and it's the major source of export revenue, contributing over $20 \%$ of the country's foreign exchange earnings. However, the productivity of coffee in Uganda is still very low estimated, at an average of $0.53 \mathrm{t} \mathrm{ha-1/yr,}$ compared to an average of $1.51 \mathrm{t} / \mathrm{ha}$ and $2.44 \mathrm{t} / \mathrm{ha}$ by Brazil and Vietnam, respectively; the leading producers of the crop (FAO, 2018). In 2016, the top five coffee producing countries worldwide included Brazil, Vietnam, Colombia, Indonesia and Ethiopia at 50.3, 24.3, 12.4, 10.7 and 7.8 million 60-kg bags, respectively; while Uganda was in the $10^{\text {th }}$ position with 3.4 million 60-kg bags (FAO, 2018). Uganda also has the lowest area under coffee production when compared to the 5 top producing countries (Brazil, $1.99 \mathrm{~m}$ ha; Vietnam, 0.60 $\mathrm{m}$ ha; Colombia, $0.87 \mathrm{~m}$ ha; Indonesia, $1.23 \mathrm{~m}$ ha; Ethiopia, $0.70 \mathrm{~m}$ ha; Uganda, $0.38 \mathrm{~m}$ ha).

Further, Uganda's coffee productivity (production per unit area; yield) is lower than that of top producers apart from Indonesia (0.52 t/ha). The apparent reason for the current low coffee production in Uganda is the low area under coffee cultivation (FAO, 2018), among other constraints (biotic and abiotic) (Olal et al., 2019; Kagezi et al., 2018a; Bukomeko et al., 2017; Paulo et al., 2010). However, the Government of Uganda (GoU) and its partners have recently embarked on increasing the area under coffee cultivation through distribution of seedlings to farmers. The current low yields are partly attributable to inappropriate agronomic practices (Olal et al., 2018; Kagezi et al., 2018b). Findings by Anim-Kwapong et al. (2010) revealed that the highest yield $(1289.5 \mathrm{~kg} / \mathrm{ha})$ of clean coffee were obtained from the highest planting density of 2667 trees/ha of Robusta coffee clones after 5 years in Ghana. However, the clones had a relatively compact growth habit, that is, smaller stem diameter and shorter internode length (Anim-Kwapong et al., 2010; Sakai et al., 2013; Salamanca-Jimenez et al., 2017). Further, an impact of tree system (multiple or single) is indispensable but a systematic investigation into such a claim is yet to be carried out. In the case of the on-going onfarm trials whose preliminary results this report highlights, we aimed to compare on-farm performance of Robusta coffee under two contrasting spacing regimes. Specifically, the second data collection was aimed at comparing the: pest incidence and damage, disease incidence and severity, and growth response and yield potential under two contrasting spacing regimes of Uganda $3 \mathrm{~m} \times 3 \mathrm{~m}$ and Brazil's $3 \mathrm{~m} \times 1 \mathrm{~m}$. The information generated will be used by researchers, policy makers and extension workers when recommending to farmers the best-bet spacing of coffee for maximum productivity. 


\section{Results}

\section{Pest incidence}

There was a significant association between RMB infestation and spacing $(x 2=11.82, d f=1, p=0.001)$. Widely spaced coffee fields were recorded with a higher RMB incidence than the closely spaced field (Fig. 1). Further, there was a significant interaction between month and spacing for incidence of $L M$ (Table 3) but non-significant for BCTB, SKL, TC, LEB, CM, Scales, BM and CBB. There was also a significant difference in incidence of pests between months for BCTB $(p<0.001)$ (Fig. 2), LM $(p<0.001)$, LEB $(p<0.001)$ and CBB $(p<0.05)$. Overall, BCTB incidence increased from $11.1 \%$ in June 2018 to17.6\% in October 2018. Incidence of LM increased from $10.5 \%$ in June 2018 to $17.6 \%$. There was a reduction in LEB incidence from 5.4\% in June 2018 to $1.9 \%$ in October 2018. The CBB incidence increased from $2.7 \%$ in June 2018 to $5.1 \%$ in October 2018.

There was a highly significant difference in pest incidence between the spacing regimes $(p<0.01)$ for all the pests except Scales $(p=0.126)$ (Table 2). The BCTB incidence was higher under close spacing of $3 \mathrm{~m} \times 1 \mathrm{~m}$ than for $3 \mathrm{~m} \times 3 \mathrm{~m}$ with a mean difference of $13.2 \%$ (Tables 1,4 and 6 ). Similarly, LEB incidence was higher under $3 \mathrm{~m} \times 1 \mathrm{~m}$ spacing than for wide widely spaced coffee, by a margin of $3.7 \%$. However, incidence of $\mathrm{LM}, \mathrm{SKL}, \mathrm{TC}, \mathrm{BM}$ and $\mathrm{CBB}$ was higher under $3 \mathrm{~m} \times 3 \mathrm{~m}$ (wide spacing) than in closely spaced coffee; with $\mathrm{CM}^{\prime}$ 's incidence being higher by $30.9 \%$ when compared to closely spaced coffee (Fig. 3).

\section{Disease incidence and severity}

No symptoms of BES were observed. There was no significant association of spacing and LRD incidence while it was significant for $\operatorname{RBD}(\chi 2=33.56, d f=1, p<0.001)$. The incidences for RBD was lower under closely spaced coffee than with widely spaced $(3 \mathrm{~m} \times 3 \mathrm{~m})$ coffee fields. Similarly, there was no significant difference in severity of LRD between spacing regimes ( $p>0.05)$. The difference in RBD between spacing regimes was very highly significant $(p<0.001)$. There was a lower RBD severity $(1.0$; no symptoms) under close spacing $(3 \mathrm{~m} \times 1 \mathrm{~m})$ compared to widely spaced $(3 \mathrm{~m} \times 3 \mathrm{~m})$ coffee whose mean severity was 1.6.

\section{Growth response and yield Growth response}

There was a significant difference in growth response between spacing regimes $(p<0.05)$ for canopy height $(\mathrm{dCAH})$ (Table 6), number of primaries (dNOP), number of stems (aNOS), leaf blade length (aLBL) and leaf blade width (aLBW). The dCAH was higher under $3 \mathrm{~m} \times 1 \mathrm{~m}$ spacing (Field 1) than for $3 \mathrm{~m} \times 3 \mathrm{~m}$ spacing (Table 7). Specifically, dCAH for closely coffee fields was higher by $7.91 \mathrm{~cm}$ and $11.84 \mathrm{~cm}$ when compared to widely spaced fields 2 and 3 , respectively (Table 9). The dNOP for $3 \mathrm{~m} \times 1 \mathrm{~m}$ and $3 \mathrm{~m} \times 3 \mathrm{~m}$ spacing was 0 and 5 , respectively. The aNOS was lower for close spacing at 2 stems/tree than wide spacing at 3 stems/tree. Similarly, dCAD was lower under close spacing than wide spacing. Further, longer leaves $(24.4 \mathrm{~cm})$ were obtained under wide spacing as compared to close spacing $(23.6 \mathrm{~cm})$ (Table 8$)$. Conversely, wider leaves were obtained under close spacing $(10.4 \mathrm{~cm})$ compared to wide spacing $(9.9 \mathrm{~cm})$.

The mean difference (d) between the close spacing field (Field 1 ) and any wide spacing field (Field 2 and Field 3 ) was positive and significant for canopy height $(d=11.84 \mathrm{~cm}$, $p=0.040$ ) (Table 9), internode length on primary $(d=0.57$, $\mathrm{p}=0.037)$ and leaf blade width $(\mathrm{d}=0.475, \mathrm{p}=0.040)$. However, $d$ was negative and significant for stem girth $(d=-0.23$, $p=0.045)$, number of primaries $(d=-6.32, p=0.003)$, number of stems $(d=-1.74, p<0.001)$, and leaf blade length $(d=-1.37$, $p=0.002$ )

\section{Green bean yield potential}

There was a significant difference in yield potential between spacing regimes $(p<0.05)$ for average yield per tree (aYT) and average yield per hectare (aYH) (Table 6). Specifically, aYH was higher under close spacing $(3 \mathrm{~m} \times 1 \mathrm{~m})$ at $5.82 \mathrm{t} \mathrm{cc} / \mathrm{ha}$ (Table 8) than wide spacing at $4.80 \mathrm{tcc} / \mathrm{ha}(3 \mathrm{~m} \times 3 \mathrm{~m})$. The aYT was however, higher under wide spacing at $2.31 \mathrm{~kg} \mathrm{cc} /$ tree than close spacing which generated $1.75 \mathrm{~kg} \mathrm{cc} /$ tree. Similarly, higher number of berries per tree was observed under wide spacing at 6,934 berries per tree than close spacing which produced 5,241 berries per tree.

The mean difference (d) for yield-related parameters between the close spacing field (Field 1 ) and any wide spacing field (Field 2 and Field 3 ) was higher and but nonsignificant for number of clusters per sample primary (Field 1 minus Field 2: $d=1.17$ berries/cluster, $p=0.230$ ) (Table 9) and yield potential per hectare (Field 1 minus Field 2: $\mathrm{d}=0.84, \mathrm{p}=0.189$; Field 1 minus Field 3 : $d=0.34, \mathrm{p}=0.609$ ). However, $d$ was negative and significant for number of active bearing primaries (Field 1 minus Field 2: $d=-11.31$, $p<0.001$ ), number of berries per cluster (Field 1 minus Field 2: $d=-7.52, p=0.001$; Field 1 minus Field $3: d=-9.60, p<0.001$ ), number of berries per tree (Field 1 minus Field 3 : $d=$ 2712.85, $p=0.003$ ) and yield per tree (Field 1 minus Field 3: $d=-0.90 \mathrm{~kg} \mathrm{cc} /$ tree, $\mathrm{p}=0.003$ ).

\section{Discussion \\ Effect of spacing on pest incidence}

Pest incidence varied with spacing with some pests exhibiting a higher incidence under close spacing $(3 \mathrm{~m} \times 1 \mathrm{~m})$ than wide spacing $(3 \mathrm{~m} \times 3 \mathrm{~m})$, and vice-versa. High RMB presence was associated with $3 \mathrm{~m} \times 3 \mathrm{~m}$ compared to $3 \mathrm{~m} \times 1 \mathrm{~m}$. It is notable that the $3 \mathrm{~m} \times 1 \mathrm{~m}$ plantation was younger than $3 \mathrm{~m} \times 3 \mathrm{~m}$ fields. RMBs are more incident when plants are stressed. The $3 \mathrm{~m} \times 3 \mathrm{~m}$ fields consisting old plants are most probably more stressed than recently established plantations. Further, RMBs require time to spread from one field to another; implying that relatively young plantations (for $3 \mathrm{~m} \times 1 \mathrm{~m}$ ) had not yet experienced high RMB populations (Mani et al., 2016). It is thus likely that as plants of closely spaced coffee mature, nutrient deficiency may set in if appropriate supplementation is not done; as RMB populations also rise; the incidence and damage by the pest can surpass that under wide spacing. Also, close spacing is highly likely to facilitate plant-to-plant RMB spread (Mani et al., 2016). Incidence of black coffee trig borer (BCTB) and leaf eating beetles (LEB) increased over time, and it was always higher under closely spaced plants. Generally, highly shaded agro-systems such as coffee encourage BCTB infestation (Bukomeko et al., 2017; Kagezi et al., 2014). Basing on the analogy by Kagezi et al. (2014), Kagezi et al. (2013) and Hultman et al. (2016), close spacing is thus suggested to have created bushy conditions which increase proximity to BCTB and LEB infested coffee plants. Notable is that both BCTB and LEB are coleopterans (Kagezi et al., 2013, 2014; Mani et al., 2016), and they can readily fly over short ranges which are created with close spacing. 
Table 1. Mean comparison for incidence of different coffee pests recorded over time, fields and spacing regimes.

\begin{tabular}{|c|c|c|c|c|c|c|c|c|c|c|c|}
\hline Month & Field & Spacing & ВСТВ & LM & SKL & TC & LEB & $\mathrm{CM}$ & Scales & BM & CBB \\
\hline \multirow{6}{*}{ Jun-18 } & Field 1 & $3 \mathrm{~m} \times 1 \mathrm{~m}$ & 20.1 & 10.9 & 1.5 & 2.5 & 8.5 & 13.5 & 0.0 & 7.5 & 0.1 \\
\hline & Field 2 & $3 \mathrm{~m} \times 3 \mathrm{~m}$ & 10.4 & 11.7 & 1.9 & 3.0 & 2.0 & 46.7 & 0.6 & 16.7 & 7.6 \\
\hline & \multirow[t]{2}{*}{ Field 3} & $3 \mathrm{~m} \times 3 \mathrm{~m}$ & 2.8 & 8.9 & 16.5 & 19.0 & 5.8 & 38.1 & 0.7 & 7.6 & 0.4 \\
\hline & & $3 \mathrm{~m} \times 1 \mathrm{~m}$ & 20.1 & 10.9 & 1.5 & 2.5 & 8.5 & 13.5 & 0.0 & 7.5 & 0.1 \\
\hline & \multirow[t]{2}{*}{ Mean } & $3 \mathrm{~m} \times 3 \mathrm{~m}$ & 6.6 & 10.3 & 9.2 & 11.0 & 3.9 & 42.4 & 0.6 & 12.2 & 4.0 \\
\hline & & Mean & 11.1 & 10.5 & 6.6 & 8.2 & 5.4 & 32.8 & 0.4 & 10.6 & 2.7 \\
\hline \multirow{5}{*}{ Oct-18 } & Field 2 & $3 \mathrm{~m} \times 3 \mathrm{~m}$ & 19.8 & 15.0 & 3.4 & 4.1 & 1.4 & 41.7 & 0.2 & 16.2 & 13.0 \\
\hline & \multirow[t]{2}{*}{ Field 3} & $3 \mathrm{~m} \times 3 \mathrm{~m}$ & 7.0 & 27.3 & 13.2 & 14.2 & 0.7 & 40.0 & 0.9 & 5.7 & 1.3 \\
\hline & & $3 \mathrm{~m} \times 1 \mathrm{~m}$ & 25.8 & 10.8 & 0.9 & 3.0 & 3.6 & 7.7 & 0.2 & 1.2 & 0.8 \\
\hline & \multirow[t]{2}{*}{ Mean } & $3 \mathrm{~m} \times 3 \mathrm{~m}$ & 13.5 & 21.1 & 8.2 & 9.1 & 1.0 & 40.8 & 0.5 & 11.1 & 7.3 \\
\hline & & Mean & 17.6 & 17.6 & 5.7 & 7.0 & 1.9 & 29.8 & 0.4 & 7.7 & 5.1 \\
\hline \multirow{5}{*}{ Mean } & Field 1 & $3 \mathrm{~m} \times 1 \mathrm{~m}$ & 22.9 & 10.8 & 1.2 & 2.8 & 6.0 & 10.7 & 0.1 & 4.4 & 0.4 \\
\hline & Field 2 & $3 \mathrm{~m} \times 3 \mathrm{~m}$ & 15.1 & 13.4 & 2.7 & 3.5 & 1.7 & 44.2 & 0.4 & 16.5 & 10.3 \\
\hline & Field 3 & $3 \mathrm{~m} \times 1 \mathrm{~m}$ & 22.9 & 10.8 & 1.2 & 2.8 & 6.0 & 10.7 & 0.1 & 4.4 & 0.4 \\
\hline & \multirow[t]{2}{*}{ Mean } & $3 \mathrm{~m} \times 3 \mathrm{~m}$ & 10.0 & 15.6 & 8.7 & 10.0 & 2.5 & 41.6 & 0.6 & 11.6 & 5.6 \\
\hline & & Mean & 14.4 & 14.0 & 6.2 & 7.6 & 3.6 & 31.3 & 0.4 & 9.2 & 3.9 \\
\hline
\end{tabular}

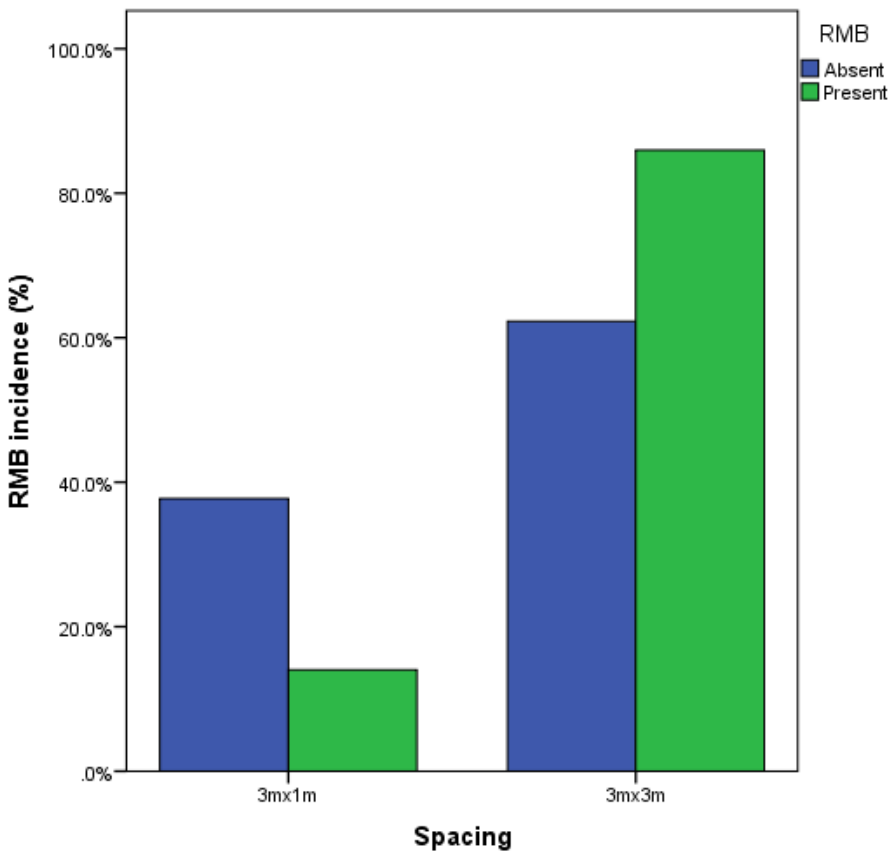

Fig 1. Relative frequency of root mealy bug under contrasting spacing regimes in Robusta coffee

Table 2. Significance of mean difference (MD) in pest incidence between two contrasting spacing regimes

\begin{tabular}{|c|c|c|c|c|c|c|c|c|c|c|}
\hline \multirow{3}{*}{ Pest } & \multirow{3}{*}{$\begin{array}{l}\text { Assumption on } \\
\text { variances }\end{array}$} & \multicolumn{2}{|c|}{$\begin{array}{l}\text { Levene's test for } \\
\text { equality of } \\
\text { variances }\end{array}$} & \multicolumn{7}{|c|}{$t$-test for equality of mean pest incidence for spacing regimes } \\
\hline & & \multirow[t]{2}{*}{$F$} & \multirow[t]{2}{*}{$p$} & \multirow[t]{2}{*}{$t$} & \multirow[t]{2}{*}{ d.f } & \multirow{2}{*}{$\begin{array}{l}p(2- \\
\text { tailed })\end{array}$} & \multirow[t]{2}{*}{ MD } & \multirow[t]{2}{*}{ S.E.D } & \multicolumn{2}{|c|}{$95 \% \mathrm{Cl}$ for $\mathrm{MD}$} \\
\hline & & & & & & & & & LL & UL \\
\hline BCTB & Non-equal variances & & & 9.791 & 199.83 & 0.000 & 12.92 & 1.319 & 10.32 & 15.52 \\
\hline LM & Equal variances & 18.16 & 0.000 & -3.024 & 320 & 0.003 & -4.82 & 1.593 & -7.95 & -1.68 \\
\hline SKL & Equal variances & 112.50 & 0.000 & -6.679 & 320 & 0.000 & -7.51 & 1.124 & -9.72 & -5.30 \\
\hline TC & Equal variances & 72.62 & 0.000 & -6.494 & 320 & 0.000 & -7.26 & 1.117 & -9.45 & -5.06 \\
\hline LEB & Equal variances & 14.50 & 0.000 & 3.634 & 317 & 0.000 & 3.50 & 0.963 & 1.60 & 5.39 \\
\hline $\mathrm{CM}$ & Equal variances & 42.65 & 0.000 & -8.864 & 319 & 0.000 & -30.97 & 3.494 & -37.84 & -24.09 \\
\hline Scales & Equal variances & 9.45 & 0.002 & -1.535 & 320 & 0.126 & -0.47 & 0.305 & -1.07 & 0.13 \\
\hline BM & Equal variances & 29.21 & 0.000 & -4.551 & 320 & 0.000 & -7.26 & 1.596 & -10.40 & -4.12 \\
\hline CBB & Equal variances & 69.81 & 0.000 & -4.890 & 319 & 0.000 & -5.17 & 1.056 & -7.24 & -3.09 \\
\hline
\end{tabular}




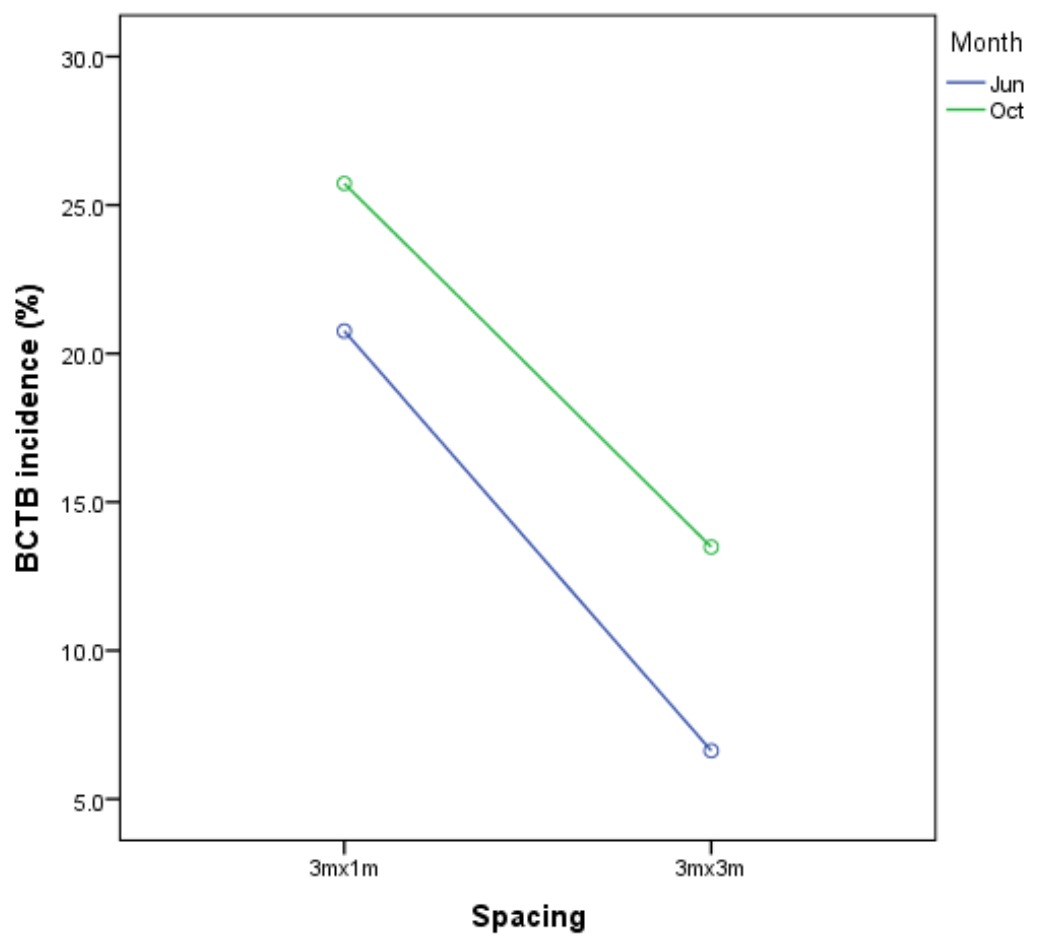

Fig 2. Variation in BCTB incidence at contrasting spacing regimes over time.

Table 3. Degrees of freedom (d.f) and mean squares for mean incidence of different coffee pests measured at two contrasting spacing regimes

\begin{tabular}{lllllllllll}
\hline Source & d.f & BCTB & LM & SKL & TC & LEB & CM & Scales & BM & CBB \\
\hline Month (M) & 1 & $3425.7^{* * *}$ & $4063.2^{* * *}$ & 67.2 & 103.4 & $953.2^{* * *}$ & 706.2 & 0.0 & 672.1 & $452.3^{*}$ \\
Spacing (S) & 1 & $11919.9^{* * *}$ & $1688.8^{* * *}$ & $4043.1^{* * *}$ & $3772.8^{* * *}$ & $895.0^{* * *}$ & $68405.2^{* * *}$ & 15.7 & $3770.5^{* * *}$ & $1920.3^{* * *}$ \\
M x S & 1 & 24.3 & $2102.6^{* * *}$ & 2.9 & 105.0 & 68.2 & 322.0 & 1.7 & 480.3 & 117.1 \\
Error & 318 & 108.4 & 163.8 & 91.1 & 89.5 & 62.4 & 872.9 & 6.7 & 180.3 & 78.3 \\
\hline
\end{tabular}

$*, * *, * * *$ significance at 5\%, 2.5 and $1 \%$ level; BCTB, black coffee trig borer; LM, leaf miners; SKL, skeletonizers; TC, tailed caterpillars; LEB, leaf eating beetles; CM, canopy mealy bugs; Scales, canopy scales; BM, coffee berry moth; CBB, coffee berry borer.

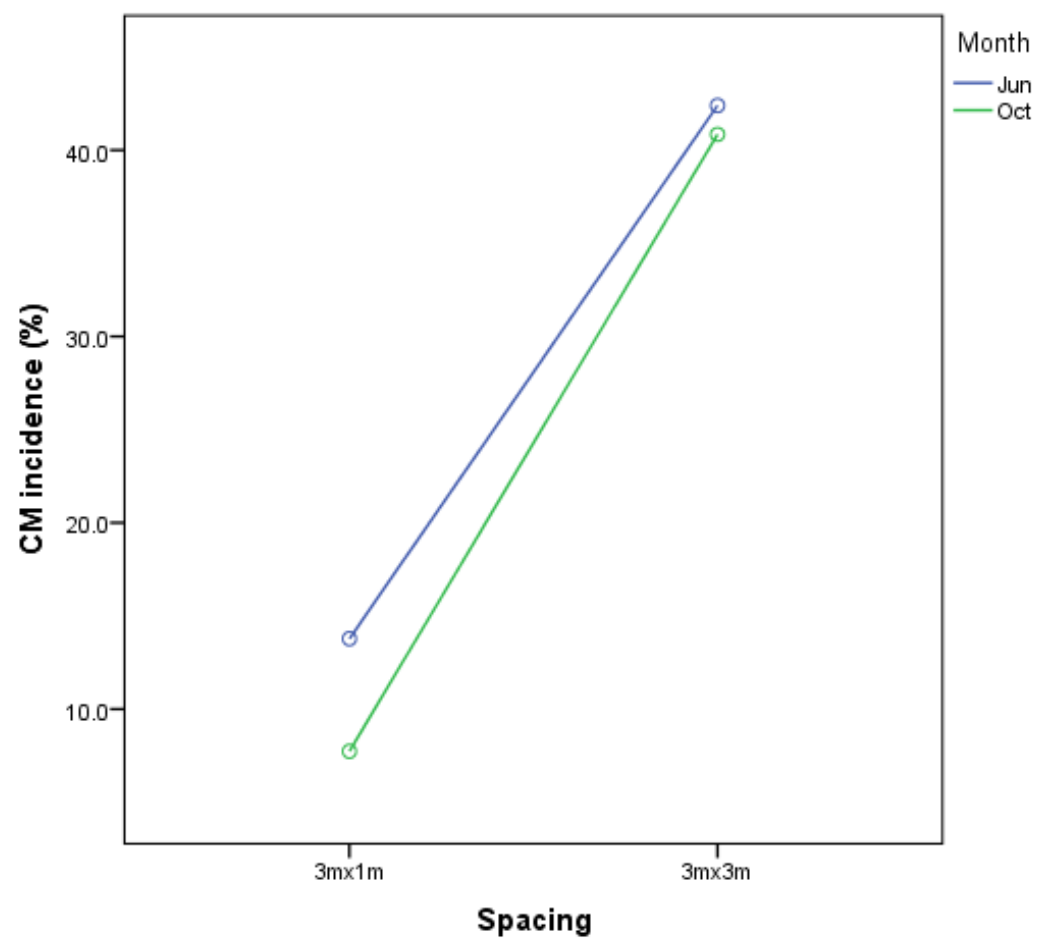

Fig 3. Variation in $\mathrm{CM}$ incidence at contrasting spacing regimes over time. 
Table 4. Mean incidence of coffee pests under two contrasting spacing regimes over time.

\begin{tabular}{|c|c|c|c|c|c|c|}
\hline \multirow[t]{2}{*}{ Pest } & \multirow[t]{2}{*}{ Month } & \multirow[t]{2}{*}{ Spacing } & \multirow[t]{2}{*}{ \% incidence } & \multirow[t]{2}{*}{ Std. error } & \multicolumn{2}{|l|}{$95 \% \mathrm{Cl}$} \\
\hline & & & & & Lower limit & Upper limit \\
\hline \multirow[t]{4}{*}{ ВСТВ } & Jun & $3 \mathrm{~m} \times 1 \mathrm{~m}$ & 20.8 & 1.48 & 17.85 & 23.67 \\
\hline & & $3 \mathrm{~m} \times 3 \mathrm{~m}$ & 6.6 & 1.00 & 4.65 & 8.60 \\
\hline & Oct & $3 \mathrm{~m} \times 1 \mathrm{~m}$ & 25.7 & 1.43 & 22.91 & 28.55 \\
\hline & & $3 \mathrm{~m} \times 3 \mathrm{~m}$ & 13.5 & 1.01 & 11.49 & 15.48 \\
\hline \multirow[t]{4}{*}{ LM } & Jun & $3 m \times 1 m$ & 10.2 & 1.81 & 6.61 & 13.72 \\
\hline & & $3 m \times 3 m$ & 10.3 & 1.23 & 7.89 & 12.72 \\
\hline & Oct & $3 \mathrm{~m} \times 1 \mathrm{~m}$ & 10.9 & 1.75 & 7.45 & 14.35 \\
\hline & & $3 \mathrm{~m} \times 3 \mathrm{~m}$ & 21.1 & 1.24 & 18.62 & 23.50 \\
\hline \multirow[t]{4}{*}{ SKL } & Jun & $3 \mathrm{~m} \times 1 \mathrm{~m}$ & 1.6 & 1.36 & -1.09 & 4.27 \\
\hline & & $3 \mathrm{~m} \times 3 \mathrm{~m}$ & 9.2 & 0.93 & 7.40 & 11.04 \\
\hline & Oct & $3 \mathrm{~m} \times 1 \mathrm{~m}$ & 0.9 & 1.32 & -1.69 & 3.51 \\
\hline & & $3 \mathrm{~m} \times 3 \mathrm{~m}$ & 8.2 & 0.93 & 6.36 & 10.04 \\
\hline \multirow[t]{4}{*}{$\mathrm{TC}$} & Jun & $3 \mathrm{~m} \times 1 \mathrm{~m}$ & 2.6 & 1.35 & -0.04 & 5.26 \\
\hline & & $3 \mathrm{~m} \times 3 \mathrm{~m}$ & 11.0 & 0.92 & 9.19 & 12.80 \\
\hline & Oct & $3 \mathrm{~m} \times 1 \mathrm{~m}$ & 2.9 & 1.31 & 0.34 & 5.49 \\
\hline & & $3 \mathrm{~m} \times 3 \mathrm{~m}$ & 9.1 & 0.93 & 7.26 & 10.90 \\
\hline \multirow[t]{4}{*}{ LEB } & Jun & $3 \mathrm{~m} \times 1 \mathrm{~m}$ & 8.6 & 1.12 & 6.39 & 10.79 \\
\hline & & $3 \mathrm{~m} \times 3 \mathrm{~m}$ & 3.9 & 0.76 & 2.41 & 5.41 \\
\hline & Oct & $3 \mathrm{~m} \times 1 \mathrm{~m}$ & 3.7 & 1.09 & 1.57 & 5.84 \\
\hline & & $3 m \times 3 m$ & 1.0 & 0.77 & -0.47 & 2.55 \\
\hline \multirow[t]{4}{*}{$\mathrm{CM}$} & Jun & $3 \mathrm{~m} \times 1 \mathrm{~m}$ & 13.8 & 4.20 & 5.51 & 22.05 \\
\hline & & $3 \mathrm{~m} \times 3 \mathrm{~m}$ & 42.4 & 2.86 & 36.77 & 48.02 \\
\hline & Oct & $3 \mathrm{~m} \times 1 \mathrm{~m}$ & 7.7 & 4.08 & -0.30 & 15.77 \\
\hline & & $3 \mathrm{~m} \times 3 \mathrm{~m}$ & 40.8 & 2.89 & 35.17 & 46.53 \\
\hline \multirow[t]{4}{*}{ Scales } & Jun & $3 \mathrm{~m} \times 1 \mathrm{~m}$ & 0.0 & 0.37 & -0.73 & 0.73 \\
\hline & & $3 \mathrm{~m} \times 3 \mathrm{~m}$ & 0.6 & 0.25 & 0.13 & 1.12 \\
\hline & Oct & $3 \mathrm{~m} \times 1 \mathrm{~m}$ & 0.2 & 0.36 & -0.50 & 0.92 \\
\hline & & $3 m \times 3 m$ & 0.5 & 0.25 & 0.02 & 1.02 \\
\hline \multirow[t]{4}{*}{$\mathrm{BM}$} & Jun & $3 \mathrm{~m} \times 1 \mathrm{~m}$ & 7.6 & 1.91 & 3.86 & 11.38 \\
\hline & & $3 \mathrm{~m} \times 3 \mathrm{~m}$ & 12.2 & 1.30 & 9.62 & 14.74 \\
\hline & Oct & $3 \mathrm{~m} \times 1 \mathrm{~m}$ & 1.1 & 1.86 & -2.59 & 4.71 \\
\hline & & $3 m \times 3 m$ & 11.1 & 1.31 & 8.47 & 13.64 \\
\hline \multirow[t]{4}{*}{ CBB } & Jun & $3 \mathrm{~m} \times 1 \mathrm{~m}$ & 0.1 & 1.26 & -2.38 & 2.57 \\
\hline & & $3 \mathrm{~m} \times 3 \mathrm{~m}$ & 4.0 & 0.86 & 2.31 & 5.68 \\
\hline & Oct & $3 \mathrm{~m} \times 1 \mathrm{~m}$ & 0.7 & 1.22 & -1.75 & 3.06 \\
\hline & & $3 \mathrm{~m} \times 3 \mathrm{~m}$ & 7.3 & 0.86 & 5.56 & 8.96 \\
\hline
\end{tabular}

Table 5. Mean difference in pest incidence at two contrasting spacing regimes.

\begin{tabular}{|c|c|c|c|c|c|c|}
\hline \multirow{2}{*}{ Pest } & \multirow{2}{*}{ I } & \multirow{2}{*}{ J } & \multirow{2}{*}{$d(I-J)$} & \multirow{2}{*}{ s.e } & \multicolumn{2}{|c|}{$95 \% \mathrm{Cl}$ for $\mathrm{d}$} \\
\hline & & & & & $\mathrm{LL}$ & UL \\
\hline ВСТВ & $3 \mathrm{~m} \times 3 \mathrm{~m}$ & $3 m \times 1 m$ & $-13.2 * * *$ & 1.25 & -15.65 & -10.72 \\
\hline LM & $3 m \times 3 m$ & $3 m \times 1 m$ & $5.2^{* * *}$ & 1.53 & 2.14 & 8.17 \\
\hline SKL & $3 m \times 3 m$ & $3 m \times 1 m$ & $7.5 * * *$ & 1.15 & 5.19 & 9.73 \\
\hline TC & $3 m \times 3 m$ & $3 m \times 1 m$ & $7.3 * * *$ & 1.14 & 5.03 & 9.52 \\
\hline LEB & $3 \mathrm{~m} \times 3 \mathrm{~m}$ & $3 m \times 1 m$ & $-3.7 * * *$ & 0.95 & -5.54 & -1.81 \\
\hline $\mathrm{CM}$ & $3 \mathrm{~m} \times 3 \mathrm{~m}$ & $3 m \times 1 m$ & $30.9 * * *$ & 3.57 & 23.85 & 37.88 \\
\hline Scales & $3 m \times 3 m$ & $3 m \times 1 m$ & 0.5 & 0.31 & -0.15 & 1.08 \\
\hline $\mathrm{BM}$ & $3 m \times 3 m$ & $3 m \times 1 m$ & $7.3^{* * *}$ & 1.62 & 4.09 & 10.47 \\
\hline CBB & $3 m \times 3 m$ & $3 \mathrm{~m} \times 1 \mathrm{~m}$ & $5.3 * * *$ & 1.07 & 3.15 & 7.35 \\
\hline
\end{tabular}

$*, * *, * * *$ significant difference $\mathrm{d}$ at $5 \%, 2.5$ and $1 \%$ level; $\mathrm{d}$, mean difference in incidence of a pest under $3 \mathrm{~m} \times 3 \mathrm{~m}$ (I) versus $3 \mathrm{~m} \times 1 \mathrm{~m}$ (J) spacing regime; $\mathrm{Cl}$, confidence interval; LL, lower limit; UL, upper limit; BCTB, black coffee trig borer; LM, leaf miners; SKL, skeletonizers; TC, tailed caterpillars; LEB, leaf eating beetles; CM, canopy mealy bugs; Scales, canopy scales; BM, coffee berry moth; CBB, coffee berry borer. 
Table 6. Significance of mean difference (MD) in growth and yield response between two contrasting spacing regimes.

\begin{tabular}{|c|c|c|c|c|c|c|c|c|c|c|}
\hline \multirow{3}{*}{$\begin{array}{l}\frac{0}{0} \\
\frac{0}{0} \\
\frac{\pi}{2} \\
>\end{array}$} & \multirow{3}{*}{$\begin{array}{l}\text { Assumption } \\
\text { variances }\end{array}$} & \multirow{2}{*}{\multicolumn{2}{|c|}{$\begin{array}{l}\text { Levene's test for } \\
\text { variances }\end{array}$}} & \multicolumn{7}{|c|}{ t-test for Equality of Means } \\
\hline & & & & \multirow[b]{2}{*}{$\mathrm{t}$} & \multirow[b]{2}{*}{ d.f } & \multirow{2}{*}{$\begin{array}{l}\mathrm{p}(2- \\
\text { tailed) }\end{array}$} & \multirow[b]{2}{*}{$\mathrm{MD}$} & \multirow[b]{2}{*}{ s.e.d } & \multicolumn{2}{|c|}{$95 \% \mathrm{Cl}$ for $\mathrm{MD}$} \\
\hline & & $\mathrm{F}$ & $\mathrm{p}$ & & & & & & $\mathrm{LL}$ & $\mathrm{UL}$ \\
\hline $\mathrm{dPH}$ & Equal variances & 18.09 & 0.000 & -0.84 & 140 & 0.402 & -3.27 & 3.894 & -10.97 & 4.43 \\
\hline dSTG & Non-equal variances & & & -1.92 & 93 & 0.058 & -0.19 & 0.100 & -0.39 & 0.01 \\
\hline $\mathrm{dCAD}$ & Equal variances & 13.83 & 0.000 & -1.92 & 140 & 0.057 & -20.32 & 10.600 & -41.27 & 0.64 \\
\hline dCAH & Equal variances & 6.30 & 0.013 & 2.38 & 140 & 0.018 & 10.08 & 4.229 & 1.72 & 18.44 \\
\hline dLLP & Non-equal variances & & & 0.87 & 113 & 0.387 & 2.85 & 3.280 & -3.65 & 9.34 \\
\hline dNOP & Non-equal variances & & & -2.73 & 93 & 0.008 & -4.73 & 1.732 & -8.17 & -1.29 \\
\hline aNOS & Equal variances & 25.88 & 0.000 & -8.04 & 140 & 0.000 & -1.31 & 0.163 & -1.63 & -0.99 \\
\hline dNAB & Non-equal variances & & & -4.82 & 128 & 0.000 & -7.97 & 1.653 & -11.25 & -4.70 \\
\hline $\mathrm{dNCP}$ & Non-equal variances & & & -1.19 & 114 & 0.237 & -0.92 & 0.776 & -2.46 & 0.62 \\
\hline $\mathrm{dNBC}$ & Non-equal variances & & & -5.87 & 96 & 0.000 & -10.52 & 1.794 & -14.09 & -6.96 \\
\hline dNIS & Non-equal variances & & & -1.12 & 106 & 0.267 & -0.92 & 0.821 & -2.54 & 0.71 \\
\hline dILS & Non-equal variances & & & 1.76 & 115 & 0.082 & 0.41 & 0.235 & -0.05 & 0.88 \\
\hline $\mathrm{dNIP}$ & Non-equal variances & & & 0.88 & 105 & 0.380 & 0.62 & 0.700 & -0.77 & 2.00 \\
\hline dILP & Non-equal variances & & & 0.31 & 127 & 0.755 & 0.06 & 0.203 & -0.34 & 0.46 \\
\hline $\mathrm{aLBL}$ & Non-equal variances & & & -2.56 & 121 & 0.012 & -0.84 & 0.326 & -1.48 & -0.19 \\
\hline aLBW & Non-equal variances & & & 2.55 & 93 & 0.012 & 0.49 & 0.191 & 0.11 & 0.87 \\
\hline aNBT & Equal variances & 10.08 & 0.002 & -2.54 & 144 & 0.012 & -1693 & 667.452 & -3012.51 & -373.97 \\
\hline aYT & Equal variances & 10.08 & 0.002 & -2.54 & 144 & 0.012 & -0.56 & 0.222 & -1.00 & -0.12 \\
\hline $\mathrm{aYH}$ & Non-equal variances & & & 2.08 & 110 & 0.040 & 1.02 & 0.491 & 0.05 & 2.00 \\
\hline
\end{tabular}

Table 7. Mean comparison for growth response and yield potential measured over spacing regimes (part 1 of 2).

\begin{tabular}{|c|c|c|c|c|c|c|c|c|c|c|c|}
\hline Field & Spacing & $\mathrm{dPH}$ & dSTG & dCAD & $\mathrm{dCAH}$ & dLLP & dNOP & aNOS & dNAB & $\mathrm{dNCP}$ & $\mathrm{dNBC}$ \\
\hline Field 1 & $3 m \times 1 m$ & 24.4 & 0.2 & 19.4 & 21.0 & 8.3 & 0 & 2 & 2 & -2 & -9 \\
\hline Field 2 & $3 m \times 3 m$ & 31.4 & 0.4 & 44.3 & 11.3 & 8.2 & 7 & 4 & 13 & -2 & 1 \\
\hline Field 3 & $3 \mathrm{~m} \times 3 \mathrm{~m}$ & 23.6 & 0.3 & 34.5 & 10.4 & 2.3 & 3 & 3 & 7 & 0 & 3 \\
\hline \multirow[t]{3}{*}{ Mean } & $3 \mathrm{~m} \times 1 \mathrm{~m}$ & 24.4 & 0.2 & 19.4 & 21.0 & 8.3 & 0 & 2 & 2 & -2 & -9 \\
\hline & $3 \mathrm{~m} \times 3 \mathrm{~m}$ & 27.7 & 0.4 & 39.7 & 10.9 & 5.4 & 5 & 3 & 10 & -1 & 2 \\
\hline & Mean & 26.5 & 0.3 & 32.4 & 14.5 & 6.5 & 3 & 3 & 7 & -1 & -2 \\
\hline
\end{tabular}

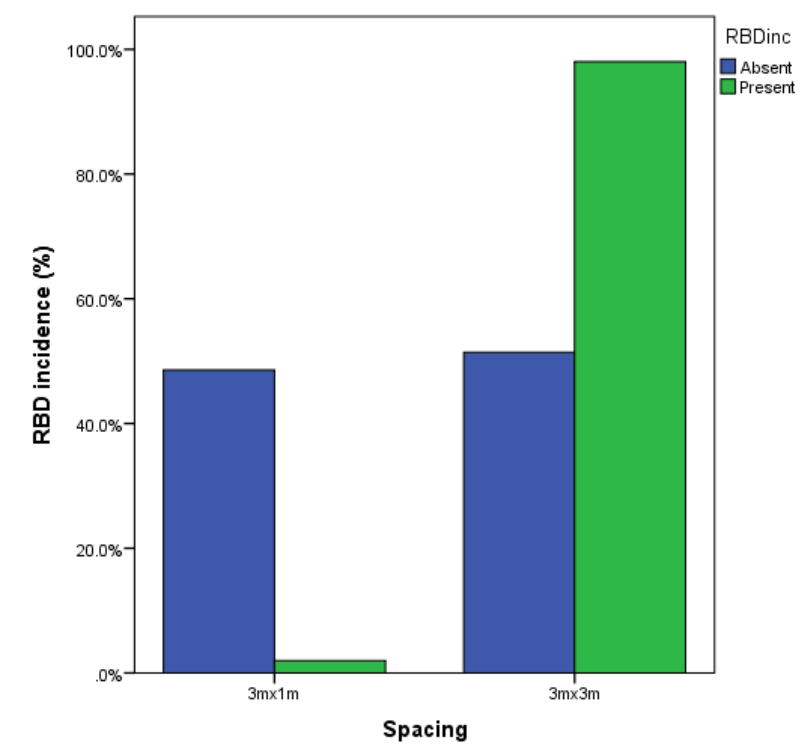

Fig 4. Variation in incidence of red blister disease under contrasting spacing regimes/ 
Table 8. Mean comparison for growth response and yield potential measured over spacing regimes (part 1 of 2).

\begin{tabular}{|c|c|c|c|c|c|c|c|c|c|c|}
\hline Field & Spacing & dNIS & dILS & dNIP & dILP & aLBL & aLBW & aNBT & aYT & $\mathrm{aYH}$ \\
\hline Field 1 & $3 \mathrm{mx} 1 \mathrm{~m}$ & 5 & 0.1 & 1 & 0.2 & 23.6 & 10.4 & 5241 & 1.75 & 5.82 \\
\hline Field 2 & $3 m \times 3 m$ & 7 & -0.2 & -1 & 0.6 & 24.1 & 9.9 & 6156 & 2.05 & 4.34 \\
\hline Field 3 & $3 \mathrm{~m} \times 3 \mathrm{~m}$ & 5 & -0.3 & 1 & -0.3 & 24.7 & 9.9 & 7799 & 2.60 & 5.32 \\
\hline \multirow[t]{3}{*}{ Mean } & $3 m \times 1 m$ & 5 & 0.1 & 1 & 0.2 & 23.6 & 10.4 & 5241 & 1.75 & 5.82 \\
\hline & $3 \mathrm{~m} \times 3 \mathrm{~m}$ & 6 & -0.3 & 0 & 0.2 & 24.4 & 9.9 & 6934 & 2.31 & 4.80 \\
\hline & Mean & 5 & -0.1 & 0 & 0.2 & 24.1 & 10.1 & 6343 & 2.11 & 5.16 \\
\hline
\end{tabular}

Table 9. Pair-wise comparison of growth and yield parameters under contrasting Robusta coffee fields for spacing.

\begin{tabular}{|c|c|c|c|c|c|c|}
\hline \multirow[t]{2}{*}{ Variable } & \multirow[t]{2}{*}{ Field $i$} & \multirow[t]{2}{*}{ Field $j$} & \multirow{2}{*}{$\begin{array}{l}\text { Mean difference }(d) d=i- \\
j\end{array}$} & \multirow[t]{2}{*}{ S.e } & \multicolumn{2}{|c|}{ 95\% confidence interval } \\
\hline & & & & & Lower limit & Upper limit \\
\hline \multirow[t]{2}{*}{$\mathrm{dPH}$} & Field 1 & Field 2 & -8.36 & 5.079 & -18.41 & 1.70 \\
\hline & & Field 3 & 0.50 & 5.289 & -9.97 & 10.98 \\
\hline \multirow[t]{2}{*}{ dSTG } & Field 1 & Field 2 & $-0.23^{*}$ & 0.113 & -0.45 & -0.01 \\
\hline & & Field 3 & -0.02 & 0.118 & -0.25 & 0.21 \\
\hline \multirow{2}{*}{ dCAD } & Field 1 & Field 2 & -27.17 & 13.815 & -54.53 & 0.18 \\
\hline & & Field 3 & -10.50 & 14.386 & -38.99 & 17.98 \\
\hline \multirow{2}{*}{ dCAH } & Field 1 & Field 2 & 7.91 & 5.465 & -2.92 & 18.73 \\
\hline & & Field 3 & $11.84^{*}$ & 5.691 & 0.57 & 23.11 \\
\hline \multirow[t]{2}{*}{ dLLP } & Field 1 & Field 2 & 1.56 & 4.426 & -7.21 & 10.32 \\
\hline & & Field 3 & 7.56 & 4.609 & -1.56 & 16.69 \\
\hline \multirow{2}{*}{ dNOP } & Field 1 & Field 2 & $-6.32^{*}$ & 2.063 & -10.40 & -2.23 \\
\hline & & Field 3 & -1.21 & 2.148 & -5.47 & 3.04 \\
\hline \multirow[t]{2}{*}{ aNOS } & Field 1 & Field 2 & $-1.74^{*}$ & 0.188 & -2.11 & -1.37 \\
\hline & & Field 3 & $-0.78^{*}$ & 0.195 & -1.17 & -0.40 \\
\hline \multirow[t]{2}{*}{ dNAB } & Field 1 & Field 2 & $-11.31^{*}$ & 2.145 & -15.56 & -7.06 \\
\hline & & Field 3 & -3.97 & 2.234 & -8.39 & 0.46 \\
\hline \multirow[t]{2}{*}{$\mathrm{dNCP}$} & Field 1 & Field 2 & 1.17 & 0.974 & -0.75 & 3.10 \\
\hline & & Field 3 & -1.83 & 1.014 & -3.83 & 0.18 \\
\hline \multirow[t]{2}{*}{$\mathrm{dNBC}$} & Field 1 & Field 2 & $-7.52^{*}$ & 2.115 & -11.71 & -3.33 \\
\hline & & Field 3 & $-9.60^{*}$ & 2.202 & -13.96 & -5.24 \\
\hline \multirow[t]{2}{*}{ dNIS } & Field 1 & Field 2 & -1.71 & 1.020 & -3.73 & 0.31 \\
\hline & & Field 3 & -0.03 & 1.062 & -2.14 & 2.07 \\
\hline \multirow[t]{2}{*}{ dILS } & Field 1 & Field 2 & 0.19 & 0.280 & -0.36 & 0.75 \\
\hline & & Field 3 & 0.23 & 0.292 & -0.35 & 0.81 \\
\hline \multirow[t]{2}{*}{ dNIP } & Field 1 & Field 2 & 1.26 & 0.827 & -0.38 & 2.90 \\
\hline & & Field 3 & -0.03 & 0.861 & -1.73 & 1.68 \\
\hline \multirow[t]{2}{*}{ dILP } & Field 1 & Field 2 & -0.29 & 0.260 & -0.80 & 0.23 \\
\hline & & Field 3 & $0.57^{*}$ & 0.270 & 0.03 & 1.11 \\
\hline \multirow[t]{2}{*}{ aLBL } & Field 1 & Field 2 & -0.80 & 0.423 & -1.64 & 0.03 \\
\hline & & Field 3 & $-1.37^{*}$ & 0.440 & -2.24 & -0.50 \\
\hline \multirow[t]{2}{*}{ aLBW } & Field 1 & Field 2 & 0.19 & 0.219 & -0.25 & 0.62 \\
\hline & & Field 3 & $0.475^{*}$ & 0.228 & 0.02 & 0.93 \\
\hline \multirow[t]{2}{*}{ aNBT } & Field 1 & Field 2 & -1585.86 & 865.985 & -3300.74 & 129.03 \\
\hline & & Field 3 & $-2712.85^{*}$ & 901.753 & -4498.56 & -927.13 \\
\hline \multirow[t]{2}{*}{ aYT } & Field 1 & Field 2 & -0.53 & 0.289 & -1.10 & 0.04 \\
\hline & & Field 3 & $-0.90^{*}$ & 0.301 & -1.50 & -0.31 \\
\hline \multirow[t]{2}{*}{$\mathrm{aYH}$} & Field 1 & Field 2 & 0.84 & 0.640 & -0.42 & 2.11 \\
\hline & & Field 3 & 0.34 & 0.666 & -0.98 & 1.66 \\
\hline
\end{tabular}

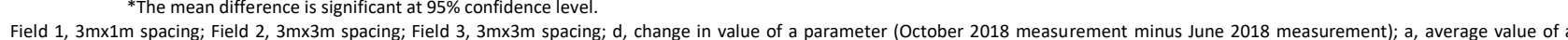
parameter (average $\left.=\frac{\text { october } 2018 \text { measurement }+ \text { fune } 2018 \text { measurement }}{2}\right) ; \mathrm{dPH}$, change in plant height $(\mathrm{cm})$; dSTG, change in stem girth $(\mathrm{cm})$; dCAD, change in canopy diameter $(\mathrm{cm})$; dCAH, change in canopy height $(\mathrm{cm})$; dLLP, change in length of longest primary $(\mathrm{cm})$; dNOP, change in number of primaries per sample stem; aNOS, average number of stems per plant; dNAB, change in number of active bearing primaries per sample stem; dNCP, change in number of clusters per sample primary; dNBC, change in number of berries per sample cluster; dNIS, change in number of internodes on sample stem; dILS, change in internode length on stem (cm); dNIP, change in number of internodes on sample primary; dILP, change in internode length on primary (cm); aLBL, average leaf blade length $(\mathrm{cm})$; aLBW, average leaf blade width $(\mathrm{cm})$; aNBT, average number of berries per tree; aYT, average yield potential per tree $(\mathrm{kg} /$ tree); aYH, average yield potential per hectare (tons cc/ha) 
Table 10. Description of growth and yield parameters measured from the Robusta coffee spacing trial.

\begin{tabular}{|c|c|c|c|c|}
\hline No & Code & Name & $\begin{array}{ll}\text { Unit } & \text { of } \\
\text { measure }\end{array}$ & Description of procedure \\
\hline 1 & PH & Plant height & $\mathrm{cm}$ & $\begin{array}{l}\text { Distance between ground level close to the plant and the tip of terminal growing point of coffee tree, measured } \\
\text { using a builder's tape }\end{array}$ \\
\hline 2 & STG & Stem girth & $\mathrm{cm}$ & Diameter of stem is taken midway the plant height, using a Vernier caliper \\
\hline 3 & CAD & Canopy diameter & $\mathrm{cm}$ & $\begin{array}{l}\text { The distance between the tips of a pair of longest primaries taken in the direction of widest separation between the } \\
\text { measured tree and neighboring trees of coffee }\end{array}$ \\
\hline 4 & CAH & Canopy height & $\mathrm{cm}$ & Distance from start of canopy to the tip of terminal growing point of coffee tree, measured using a builder's tape \\
\hline 5 & LLP & $\begin{array}{l}\text { Length of longest } \\
\text { primary }\end{array}$ & $\mathrm{cm}$ & The distance from the stem to the tip of longest primary \\
\hline 6 & NOP & Number of primaries & count & Counting all the primaries on a sample stem \\
\hline 7 & NOS & Number of stems & count & Counting all the stems on a coffee tree \\
\hline 8 & NAB & $\begin{array}{l}\text { Number of active } \\
\text { bearing primaries }\end{array}$ & count & Counting all the primaries containing a cherry, berry or pinheads on a sample stem \\
\hline 9 & NCP & $\begin{array}{l}\text { Number of clusters per } \\
\text { primary }\end{array}$ & count & Counting all the clusters on the sample primary \\
\hline 10 & NBC & $\begin{array}{l}\text { Number of berries per } \\
\text { cluster }\end{array}$ & count & Counting all the berries on a sample cluster of a sample primary \\
\hline 11 & NIS & $\begin{array}{l}\text { Number of internodes } \\
\text { per stem }\end{array}$ & count & Counting all the internodes on stem \\
\hline 12 & ILS & $\begin{array}{l}\text { Internode length per } \\
\text { stem }\end{array}$ & $\mathrm{cm}$ & $\begin{array}{l}\text { Distance between two internodes on sample stem, obtained by dividing plant height by number of internodes on } \\
\text { stem }\end{array}$ \\
\hline 13 & NIP & $\begin{array}{l}\text { Number of internodes } \\
\text { per primary }\end{array}$ & count & Counting all internodes on longest primary \\
\hline 14 & ILP & $\begin{array}{l}\text { Internode length on } \\
\text { primary }\end{array}$ & $\mathrm{cm}$ & $\begin{array}{l}\text { Distance between two internodes on sample primary, obtained by dividing length of longest primary by number of } \\
\text { internodes on primary }\end{array}$ \\
\hline 15 & LBL & Leaf length $(\mathrm{cm})$ & $\mathrm{cm}$ & $\begin{array}{l}\text { Distance between point of attachment of leaf blade on leaf stalk and the apex of the leaf, measured using a foot } \\
\text { ruler }\end{array}$ \\
\hline 16 & LBW & Leaf width $(\mathrm{cm})$ & $\mathrm{cm}$ & Distance between the extremes of a widest part of the leaf blade, measured using a foot ruler \\
\hline 17 & NBT & Number berries per tree & count & $\begin{array}{l}\text { Multiplying the number of active bearing primaries, number of clusters per primary, and number of berries per } \\
\text { cluster }\end{array}$ \\
\hline 18 & NBH & $\begin{array}{l}\text { Number of berries per } \\
\text { hectare }\end{array}$ & count & $\begin{array}{l}\text { Multiplying the number of active bearing primaries, number of clusters per primary, number of berries per cluster, } \\
\text { and number of coffee plants in a hectare. Plant population }=10,000 / \text { spacing }\end{array}$ \\
\hline 19 & YT & Estimated yield per tree & $\mathrm{kg} \mathrm{cc} /$ tree & $\begin{array}{l}\text { Multiplying number of berries per tree with weight of coffee green. It is assumed that } 1 \mathrm{~kg} \text { of cherries contains about } \\
600 \text { cherries; and that } 1 \mathrm{~kg} \text { of clean coffee }(\mathrm{cc}) \text { can be obtained from } 5 \mathrm{~kg} \text { of cherries. The yield per tree is recorded in } \\
\mathrm{kg} \mathrm{cc} / \text { tree. }\end{array}$ \\
\hline 20 & YH & Estimated yield per ha & tons $\mathrm{cc} / \mathrm{ha}$ & $\begin{array}{l}\text { Multiplying number of berries per hectare with weight of coffee green. It is assumed that } 1 \mathrm{~kg} \text { of cherries contains } \\
\text { about } 600 \text { cherries; and that } 1 \mathrm{~kg} \text { of clean coffee (cc) can be obtained from } 5 \mathrm{~kg} \text { of cherries. The yield per hectare is } \\
\text { recorded in tons } \mathrm{cc} / \mathrm{ha} \text {. }\end{array}$ \\
\hline
\end{tabular}

thus, infesting new plants. Subject to further investigation, close spacing could be a remedy for $L M, S K L, T C, B M$ and CBB whose incidence was lower under wide spacing than with close spacing.

\section{Effect of spacing on disease incidence and damage}

Absence of symptoms for some diseases (like BES and RBD) under close spacing is attributable to absence of source of inoculums as the plantation was relatively young. The RBD severity being higher under wide spacing than close spacing fields is attributable to accumulated inoculums (Waller et al., 2007) for RBD as the field had been bearing for over 10 years. LRD equally affected both closely spaced and widely spaced coffee fields. This trend of symptom expression requires to be monitored across seasons (wet and dry seasons). In the current study, data was collected in a rainy period of June and October 2018.

\section{Effect of spacing on growth response}

Competition among plants drives their relative response for specific growth parameters under contrasting spacing regimes (Anim-Kwapong et al., 2010; Bell et al., 1995; Canell, 1985; DaMatta et al., 2007). Under high plant density (close spacing), plants tend to compete for sunlight as facilitated by hormonal distribution. As a consequence, abnormal elongation of stems under close spacing arises (Kiup, 2017; Maddonni and Martínez-Bercovich, 2014; Rakocevic et al., 2018); as witnessed with high canopy height and relatively low increase in STG. Conversely, widely spaced coffee plants can access radiation from all directions with minimum competition; accounting for higher increase in lateral growth (high increase in CAD) than close spacing. The high increase in CAD is also explained by the high likelihood for multiple stems per plant whose growth habit ultimately widens the coffee tree canopy (DaMatta et al., 2007; Anim-Kwapong et al., 2010). To corroborate the view on CAD changes, this study documented a higher number of stems per plant under wide spacing than close spacing. Similar to the trend on CAD and CAH, LBL was higher under close spacing while LBW was higher under wide spacing. It is suggested that close spacing creates a lot of competition among Robusta coffee trees which compromises on their robustness attributes (Sakai et al., 2013; Salamanca-Jimenez). However, a follow-up detailed study under complete control of planting dates and blocking for variation management is necessary for solid recommendations.

\section{Effect of spacing on yield potential}

High aYT under wide spacing did not translate into better $\mathrm{aYH}$. The highest yield potential was obtained under close spacing. The observation suggests that as plant density increases, yield increases as earlier suggested by AnimKwapong et al. (2010) and Paulo et al. (2010). It is imperative that in-depth analysis of relationships among pest incidence, disease incidence and severity, growth response and yield in coffee is conducted. So far, BCTB incidence is shown to be higher under close spacing than wide spacing yet this study also suggests that better yield is achievable as plant density is increased. Unless the pests and diseases whose incidence and severity increases with plant density are sustainably managed (Bell et al., 1995; Canell, 1985; DaMatta et al., 2007; Paulo et al., 2010), the apparent yield increase under high plant densities may not be realized by farmers.

\section{Materials and methods}

\section{Plant materials}

Clonal cuttings of coffee wilt disease resistant (CWD-r) Robusta coffee varieties were used. The planting materials, a composite of seven CWD-r varieties were obtained from National Coffee Research Institute (NaCORI). NaCORI is a semi-autonomous public research institute mandated to conduct research on coffee and cocoa in Uganda under 
policy guidance of Ministry of Agriculture, Animal Industry and Fisheries through National Agricultural Research Organisation (www.naro.go.ug).

\section{Study site}

This study was imposed in July 2017 on already established farmer's Robusta coffee fields at Bunjako Island, Buwama sub-county in Mpigi district. The Island lies at an altitude of 1138 m.a.s.I and latitude/longitude of $00.2778 / 32.134444$ decimal degrees $\left(00^{\circ} 10^{\prime} 10^{\prime \prime} \mathrm{N} / 32^{\circ} 08^{\prime} 04^{\prime \prime}\right.$ E degrees, minutes and seconds). Mpigi district experiences a bi-modal rainfall pattern with first rains occurring between March and May and second rains coming between September and November with an average rainfall amount of $1320 \mathrm{~mm}$ though in many areas around the Lake zone (including Bunjako Island) it is between $1750 \mathrm{~mm}$ and $2000 \mathrm{~mm}$ (www.mpigi.go.ug/about/geographical-features).

\section{Experimental design}

Three fields were selected to constitute the experiment namely Field 1 , Field 2 and Field 3 containing coffee plants spaced at $3 \mathrm{~m} \times 1 \mathrm{~m}, 3 \mathrm{~m} \times 3 \mathrm{~m}$ and $3 \mathrm{~m} \times 3 \mathrm{~m}$, respectively. Field 1 is located near the lake (Bunjako Island) and it is a coffee system. Field 2 is also located near the lake at Bunjako Island but it is a coffee-agroforestry system. Field 3 is located some $3 \mathrm{~km}$ away from the lake (host farmer's home); the field is a coffee-banana-agroforestry system). Coffee trees in Field 1 were about 3 years old, trees in Field 2 were about 13 years old but had been stumped and the rejuvenated trees were about 3 years old. Coffee trees in Field 3 were about 8 years old. Two replicates were sampled in the field where coffee trees are spaced at $3 \mathrm{~m} \times 1 \mathrm{~m}$, each replicate having 3 plots of 9 coffee trees each and a total of 54 trees. In fields 2 and 3 where coffee trees are spaced at $3 \mathrm{~m} \times 3 \mathrm{~m}$, there is 1 plot in a block (replicate) and 18 coffee plants were sampled from the plot for three blocks, giving a total of 54 coffee plants per field.

\section{Data collection}

\section{Pests' incidence}

The selected coffee trees were assessed for root mealybugs (RMB), black coffee twig borers (BCTB), leaf miners (LM), leaf skeletonizers (SKL), leaf eating beetles (LEB), canopy scales (Scales), canopy mealybugs (CM), tailed caterpillars (TC), berry moths (BM), and berry borer (CBB). Trees were assessed for presence or absence of RMB basing on either evidence of ant activity and/or whitish powdery-like materials around the collar region. For BCTB, the total number of primary branches and those infested by BCTB on the sample stem were counted and percentage of infested primary branches(twigs) was computed (infested/total) $\mathrm{x}$ 100). To assess LM, SKL, TC and LEB, the bearing head of the coffee tree was divided into three imaginary sections upper, middle and lower. One primary branch with berries was randomly selected from each section, from which coffee leaves, berry clusters and berries were sampled for assessment. The total number of leaves as well as those damaged by LM, SKL, TC, and LEB was recorded. The number of coffee clusters as well as those infested with CMB, Scales, and $\mathrm{BM}$ sampled twig were also recorded. From a middle cluster of the twig, the number of berries and those infested with $\mathrm{CBB}$ were also recorded.

\section{Diseases incidence and severity}

Incidence (presence or absence) and severity (scale of 1-5, where $1=$ no disease and $5=>50 \%$ of leaves infected) were scored for coffee leaf rust (LRD) (fungal causal agent, Hemileia vastatrix), red blister disease (Cercospora coffeicola) (RBD) and brown eye spot (C. coffeicola) (BES).

\section{Vegetative growth response}

Thirteen growth response variables were measured per coffee tree (Table 1). These variables include plant height, stem girth, canopy diameter, canopy height, length of primary, number of primaries, number of stems per tree, number of internodes per stem, internode length per stem, number of internodes per primary, internode length on primary, leaf blade length and leaf blade width.

\section{Yield potential}

Seven yield-related variables were measured per tree (Table 10). The variables include number of active bearing primaries, number of clusters per primary, number of berries per cluster, number of berries per tree, number of berries per hectare, yield potential per tree and yield potential per hectare.

\section{Data analysis}

The RMB was recorded as a binary variable ( 1 for presence, 0 for absence) and a chi-square test was carried out in order to ascertain if pest incidence is associated with spacing regime at a significance level of $5 \%(p<0.05)$. For rest of pests scored, an independent samples two-tailed $t$-test at $p<0.05$ preceded by Levene's F-test for equality of variances at $p<0.05$ and comparison for mean pest incidence was conducted using IBM SPSS Statistics v21. This $t$-test enabled comparison of mean pest incidence under the contrasting spacing regimes; $3 \mathrm{~m} \times 1 \mathrm{~m}$ and $3 \mathrm{~m} \times 3 \mathrm{~m}$, irrespective of month of data collection and age (field) of coffee plantation.

In order to ascertain if there is a temporal (month of data collection) effect on observed differences in pest incidence between spacing regimes, an analysis of variance (ANOVA) for unbalanced treatment structure was conducted using the following general linear model:

$$
y_{i j k}=\mu+M_{i}+S_{j}+M S_{k}+e_{i j k}
$$

Where; $y_{i j k}$ is any observed pest incidence, $\mu$ is grand mean for pest incidence, $M_{i}$ is the $i^{t h}$ month of data collection, $S_{j}$ is the $j^{\text {th }}$ spacing regime, $M S_{k}$ is the $k^{\text {th }}$ month by spacing interaction, and $e_{i j k}$ is random error. The decision on significant differences in pest incidence across months and spacing regimes was made at $95 \%$ confidence level.

\section{Disease incidence}

Like for pest incidence, the $t$-test at $p<0.05$ preceded by Levene's F-test for equality of variances at $\alpha=5 \%$ and comparison for mean disease incidence was conducted to compare of mean disease incidence under the two spacing regimes.

The $t$-test was followed by ANOVA (F-test) at $\mathrm{p}<0.05$ for unbalanced treatment structure in order to ascertain for interactive effects of time (month of data collection) and spacing on incidence of coffee diseases using a similar model:

$$
y_{i j k}=\mu+M_{i}+S_{j}+M S_{k}+e_{i j k}
$$


where $y_{i j k}$ is any observed disease incidence, $\mu$ is grand mean for disease incidence, $M_{i}$ is the $i^{\text {th }}$ month of data collection, $S_{j}$ is the $j^{\text {th }}$ spacing regime, $M S_{k}$ is the $k^{\text {th }}$ month by spacing interaction, and $e_{i j k}$ is random error. The decision on significant differences in disease incidence across months and spacing regimes was made at $95 \%$ confidence level.

\section{Growth response and yield potential}

Growth was measured as a change (d) in each of the parameters detailed in Table 1 from June 2018 to October 2018. The number of stems per tree (NOS), leaf blade length (LBL) and leaf blade with (LBW) were expressed as average (a) of the June 2018 and October 2018 records, hence aNOS, aLBL and aLBW, respectively.

For each of June 2018 and October 2018, yield potential per tree ( $\mathrm{kg} \mathrm{cc} /$ tree) and yield per hectare (tons $\mathrm{cc} / \mathrm{ha}$ ) were estimated using number of active bearing primaries (NAB), number of clusters per primary (NCP), number of berries per cluster (NBC), cherry weight and shrinkage factor (SF) from cherries to green or clean coffee (cc) as follows:

$N B T=N A B * N C P * N B C$

$N B H=N A B * N C P * N B C *$ plant population

$Y T=N B T *$ cherry weight $/ S F=(N B T / 600) / 5$.

$Y H=Y T *$ plant population

NBT, NBH, YT and YH stand for number of berries per coffee tree, number of berries per hectare, yield potential per tree ( $\mathrm{kg} \mathrm{cc} /$ tree) and yield potential per hectare (t cc/ha), respectively. It was assumed that $1 \mathrm{~kg}$ of cherries contains about 600 cherries and that $1 \mathrm{~kg}$ of clean coffee (cc) can be obtained from $5 \mathrm{~kg}$ of cherries (hence shrinkage ratio of $5: 1$ ). The plant population is given by:

Plant population (trees $/$ ha $)=10,000 /($ spacing $)$; hence $10,000 /(3 * 1)$ and $10,000 /(3 * 3)$ for $3 \mathrm{~m} \times 1 \mathrm{~m}$ and $3 \mathrm{~m} \times 3 \mathrm{~m}$ spacing, respectively.

After obtaining yield-related traits for June and October, average values were calculated as follows:

$a N B T=\frac{N B T \text { for October }+N B T \text { for June }}{2}$
$a Y T=\frac{Y T \text { for October }+Y T \text { for June }}{2}$
$a Y H=\frac{Y H \text { for October }+Y H \text { for June }}{2}$

An independent samples two-tailed $t$-test at $p<0.05$ preceded by Levene's F-test for equality of variances at $p<0.05$ and comparison for mean pest incidence was then conducted using IBM SPSS Statistics V21. This $t$-test enabled comparison of growth and yield response under the contrasting spacing regimes; $3 \mathrm{~m} \times 1 \mathrm{~m}$ and $3 \mathrm{~m} \times 3 \mathrm{~m}$. This was followed by a Bonferroni test for pair-wise comparison in growth response and yield across fields. The differences between any two fields for a parameter were declared significant at $95 \%$ confidence level.

\section{Conclusion}

Plant density has a significant effect on pest incidence, disease incidence and severity, growth response and yield of Robusta coffee. The plant density effect also has temporal dimensions. As plant stress increases under close spacing pest problems such as RMB and BCTB also increase. Similarly, high yield potential per tree triggers plant stress due to increase in soil water and nutrient harvesting, resulting in disease challenges especially RBD. The growth robustness in Robusta coffee cannot be fully expressed under close spacing though this notion requires further optimization. It is our view that whereas yield potential is high at high tree densities, a detailed longitudinal analysis on the achievability of this apparent yield potential amidst pest, disease and abiotic constraints is conducted as basis for solid recommendation to farmers.

\section{Acknowledgment}

This activity was funded at National Coffee Research Institute (NaCORI) by Uganda Coffee Development Authority (UCDA)

\section{Conflict of interest}

The authors have no conflict of interest to declare.

\section{References}

Anim-Kwapong G, Anim-Kwapong E, Oppong F (2010) Evaluation of some robusta coffee (Coffea canephora pierre ex a. Froehner) clones for optimal density planting in Ghana. Afr J Agr Res. 5:84-89.

Bell M, Fischer RA, Byerlee D, Sayre K (1995) Genetic and agronomic contributions to yield gains: A case study for wheat. Field Crops Res. 44:55-65.

Bukomeko $\mathrm{H}$, Jassogne L, Kagezi GH, Mukasa D, Vaast $P$ (2017) Influence of shaded systems on Xylosandrus compactus infestation in Robusta coffee along a rainfall gradient in Uganda. Agr Forest Entomol. 1:1-7.

Cannell MG (1985) Dry matter partitioning in tree crops, in: Attributes of Trees as Crop Plants. NERC Open Research Archive, bbotts Ripton, Institute of Terrestrial Ecology, pp. 160-193.

DaMatta F, Ronchi C, Maestri M, Barros R (2007) Ecophysiology of coffee growth and production. Braz J Plant Physiol. 19:485-510.

FAO (2018) Green coffee production for 2016. Food and Agriculture Organization of the United Nations, Rome, Italy.

Hultman C (2016) Black Coffee Twig Borer, Xylosandrus compactus (Eichhoff) on robusta coffee in Uganda Impact of shade level on BCTB and knowledge levels about BCTB. Swedish University of Agricultural Sciences, Uppsala, Sweden. 78 pages.

Kagezi GH, Kucel P, Kobusinge J, Olango N, Nakibuule L Wagoire WW (2018a) Predicting the response of insect pests and diseases of Arabica coffee to climate change along an altitudinal gradient in Mt. Elgon region, Uganda. J Agric Environ Sci. 7(1):134-140.

Kagezi GH, Kucel P, Kobusinge J, Olango DN, Nakibuule L, Nanjego W, Nambozo PB, Olal S, Wagoire WW (2018b) Farmers' knowledge and perception of the use of pesticides in Arabica coffee, Coffea arabica agro-ecologies of Uganda. J Agric Environ Sci. 7(2):173-188.

Kagezi GH, Kucel P, JP, Egonyu JP, Ahumuza G, Nakibuule L, Kobusinge J, Wagoire WW (2014) Implications of Black Coffee Twig Borer on cocoa in Uganda. Uganda J Agric Sci. 15(2):179-189. 
Kagezi GH, Kucel P, Egonyu JP, Nakibuule I, Kobusinge J, Ahumuza G, Matovu R, Nakendo S, Luzinda H, Musoli CP, Kangire A, Chesang BF (2013) Impact of the black coffee twig borer and farmers' coping mechanisms in Uganda. African Crop Science Conference Proceedings 11:285-292.

Kiup E (2017) Maximizing nutrient utilisation and soil fertility in smallholder coffee and food garden systems in Papua New Guinea by managing nutrient stocks and movement (MPhil). James Cook University, Queensland, Australia.

Maddonni G, Martínez-Bercovich J (2014) Row spacing, landscape position, and maize grain yield. Int J Agron. 2014:1-12.

Mani M, Smitha MS, Najitha U (2016) Root mealybugs and their management in horticultural crops in India. Pest Management in Horticultural Ecosystems 22(2):103-113.

Olal S, Bitalo DN, Olango ND, Mulindwa J, Ochwo S, Opiyo SO, Arinaitwe G, Ogwok E (2019) De novo genome sequence of a Fusarium xylarioides race pathogenic to robusta coffee (Coffea canephora) in Uganda. Microbiol Resour Announc. 8:e00520-19.
Paulo E, Furlani JrE (2010) Yield performance and leaf nutrient levels of coffee cultivars under different plant densities. Sci Agr. 67:720-726.

Rakocevic M, Dos Santos MB, Kitzberger CS (2018) Berry distributions on coffee trees cultivated under high densities modulate the chemical composition of respective coffee beans during one biannual cycle. Int J Fruit Sci. 18:117-137.

Sakai E, Barbosa EA, Silveira JM, Pires RC (2013) Coffea arabica (cv catuaí) production and bean size under different population arrangements and soil water availability. Eng Agr Jaboticabal. 33:145-156.

Salamanca-Jimenez A, Doane T, Horwath W (2017) Nitrogen use efficiency of coffee at the vegetative stage as influenced by fertilizer application method. Front Plant Sci. 8:1-11.

Waller JM, Bigger M, Hillocks RJ (2007) Coffee pests, diseases and their management. CABI International. 434 pages. 